\section{Corneal toxicity: the epithelium and stroma in iatrogenic and factitious disease}

J Dart

Keywords: toxicity; keratoconjunctivitis; cornea; iatrogenic; factitious

Introduction

A toxic substance can be defined as one which by its chemical action causes damage to structure, or a disturbance of function, over and above any therapeutic effect. Classification systems for corneal toxicity are by disease, route of exposure and time course, or by agent ${ }^{1,2}$ (see Table 1). The cornea may be involved alone or with the conjunctiva. Corneal toxicity is caused by chemical trauma, for which diagnosis is seldom a problem, and also by iatrogenic and factitious disease ${ }^{3}$ (intentional disease production by the patient), which are both often overlooked. This review will focus on iatrogenic and factitious corneal toxicity, and its diagnosis, epidemiology, pathogenesis, prediction, and assessment of toxicity, treatment and prevention.

\section{Diagnosis of toxicity}

Iatrogenic toxicity occurs in patients with acute or chronic ocular surface disorders as a result of both the short-term and, more often, the longterm use of topical medications. ${ }^{4,5}$ The clinical signs of both iatrogenic and factitious disease are usually nonspecific and identical to those resulting from other causes of corneal epithelial disease such as punctate keratopathy, coarse focal keratopathy, pseudodendrites, filamentary keratopathy, and persistent epithelial defect. More specific signs occur with the preservative phenylmercuric nitrate toxicity, ${ }^{1}$ which may produce band keratopathy. Other unique reactions include cumulative drug deposition, the keratopathy of anaesthetic abuse, and thiomersal keratoconjunctivitis. Cumulative drug deposition is because of systemic medications (amiodarone, indomethacin, 
Table 1 Classification systems for corneal toxicity

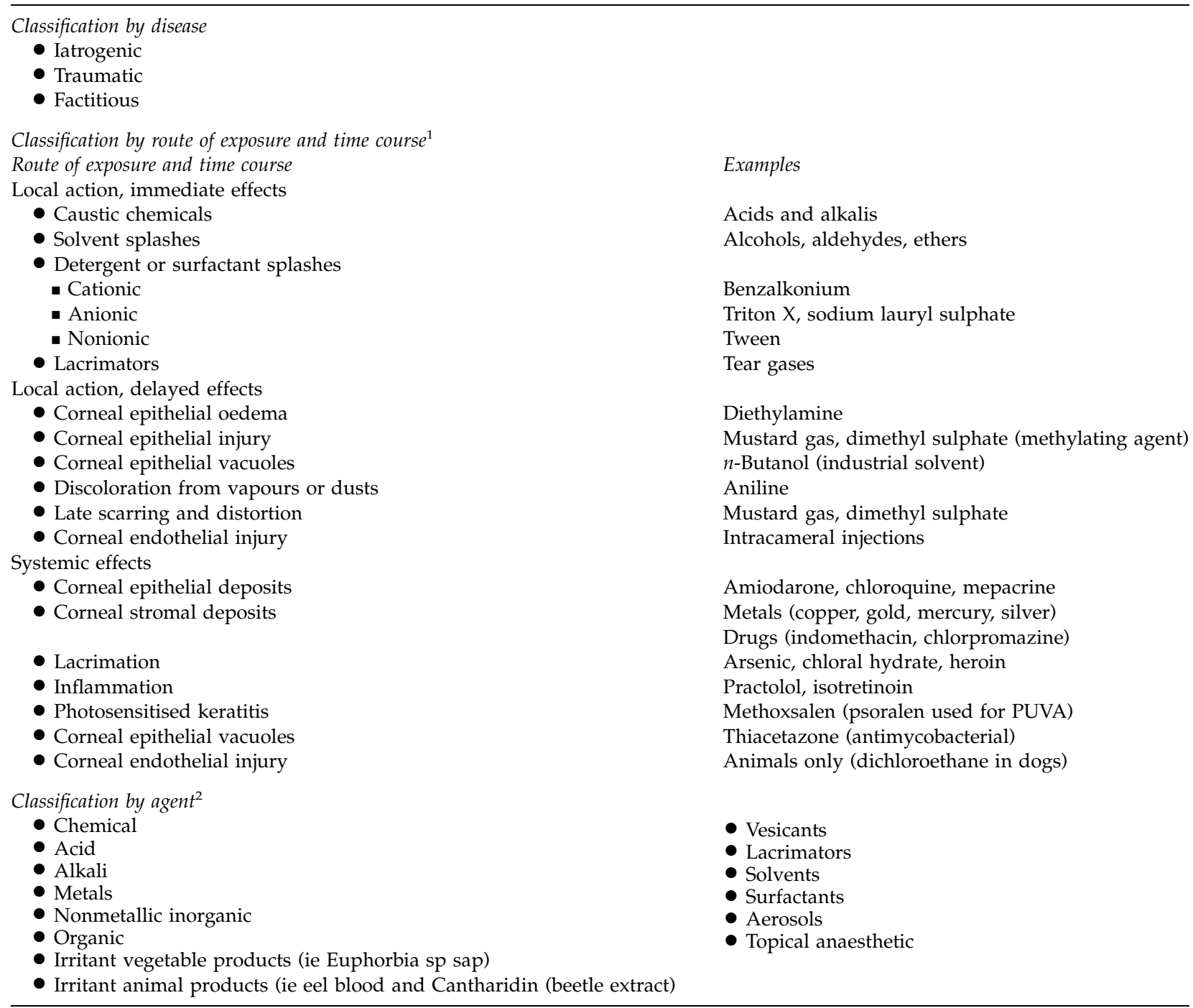

chloroquine) causing deposits within the corneal epithelium $^{1}$ that are usually of little functional significance. Topical anaesthetic abuse initially results in nonspecific clinical features of toxicity but, when fully developed, has the characteristic findings of coalescent corneal infiltrates, iritis, a Wessely ring, and hypoyon. ${ }^{6}$ Thiomersal keratoconjunctivitis produces a superior corneal punctate keratopathy, neovascularisation, corneal opacity and, in a few advanced cases, corneal epithelial failure. ${ }^{7}$

Factitious disease is usually either as a result of the abuse of topical preparations or from mechanical trauma. The topical anaesthetics and the more toxic antivirals (idoxuridine and trifluorothymidine) are among the more commonly abused drops. ${ }^{6}$ Mucous fishing syndrome $^{8}$ and congenital or acquired corneal anaesthesia, ${ }^{9}$ particularly in children, can be mistaken for factitious disease and should be considered in the differential diagnosis of patients with these clinical signs.

Corneal toxicity, with the exception of cumulative drug deposition, is associated with variable degrees of conjunctival involvement. These changes vary from a subtle punctate stain with Rose Bengal, a papillary, follicular or mixed follicular and papillary conjunctivitis with or without scarring, or conjunctival erosions.

\section{Epidemiology}

In tertiary referral centers, iatrogenic disease is either a common cause or an exacerbating factor in ocular surface disorders. A comprehensive clinical study of this problem was carried out 20 years ago and, although drug 
Table 2 Summary of findings from Wilson ${ }^{4}$

Study location: a tertiary referral centre

Study design: consecutive case series

Case definition: cases were classified as drug reactions if they were (i) unanticipated, unrecognised, or misunderstood by the referring ophthalmologist, (ii) clinically unacceptable in terms of the underlying diagnosis, and (iii) clinically important Results:

- 134/1024 (13.09\%) of a consecutive series fulfilled the case definition

- All patients had conjunctival reactions of which the commonest were papillary, follicular, or delayed hypersensitivity

- Corneal involvement occurred in 119/134 of which 38 were confluent or persistent epithelial defects

- Resolution of toxicity

- Days to improve: 2-49 (median 9), $n=52$

- Days to resolve: 7-93 (median 28.5), $n=26$

- Associated epidemiological factors:

- The number and variety of treating practitioners (optoms, etc)

- The number of practitioners consulted

- The number of practitioners who treated

- Specific ophthalmologist ( $8 \%$ treated $40 \%$ of drug-related disease)

- Number of days treated (mean 247 days for drug reactions (DR) vs 67 days for inflammatory disease controls (ID)

- Number of medications (median 4.5 for DR vs 2.5 for ID)

- Symptoms of OSD, lid and periorbital disease greater

- Drugs and preservatives

- Drugs: idoxuridine, arabinoside A, aminoglycosides, pilocarpine, chloramphenicol, multiple Rx

- Preservatives: benzalkonium chloride, phenylmercuric nitrate, thiomersal, EDTA

- Underlying diagnoses (over $50 \%$ inactive at the time of diagnosis)

- KCS, HSV, postherpetic ulcer, no other diagnosis and glaucoma in $60 \%$ of cases - 62 other diagnoses responsible for the rest,

- Inaccuracy of referring ophthalmologist's diagnosis (47\% for DR vs $64 \%$ for ID)

- Dry eyes for toxic papillary conjunctivitis

Conclusions

- Drug reaction was the second most common diagnosis in this tertiary referral centre

- Patients with dry eyes are particularly at risk

- Idoxuridine and benzalkonium in artificial tears were the two most common precipitating drugs

- The time taken for improvement and resolution of drug reactions may be prolonged

usage has changed, it remains a very useful account of the epidemiology of the iatrogenic effects of topical medications. ${ }^{4,5}$ Table 2 summarises the findings of this study, which was conducted in a tertiary referral centre, where $134 / 1024(13.09 \%)$ of consecutive cases were classified as drug reactions. ${ }^{1}$ Corneal involvement occurred in 119/134 (88\%) of these cases and 38 (28\%) had frank corneal epithelial defects or indolent ulcers. The commonest conjunctival reactions were toxic papillary, toxic follicular, and delayed hypersensitivity. The commonest associated drugs were idoxuridine (IDU), arabinoside A, aminoglycosides, pilocarpine, chloramphenicol, and the preservatives benzalkonium chloride, phenylmercuric nitrate (which is no longer used in the UK), thiomersal, and EDTA. Over $50 \%$ of the underlying conditions were inactive at the time of diagnosis of ocular surface toxicity. Over half the cases either had no diagnosis or had dry eye, herpes simplex virus keratitis, or glaucoma. Another 62 diagnoses accounted for the remaining cases. There were several practitioner factors associated with iatrogenic toxicity, which are summarised in Table 2. Of particular relevance are the number of medications used and the prolonged treatment period before diagnosis in the iatrogenic toxicity group. Healing was prolonged with patients taking 2-49 (median 9) days to improve and 7-93 (median 28.5) days to resolve.

These data reflect current experience with topical drug toxicity, although topical acyclovir, where it is available, has reduced the use of the more toxic antivirals arabinoside A and trifluorothymidine. Toxicity remains a problem with the new glaucoma medications. Benzalkonium is still the principal preservative in the majority of topical preparations, whereas thiomersal and phenylmercuric nitrate are now seldom used.

\section{Pathogenesis}

Mechanisms of pathogenesis vary and, although understanding these is not important for diagnosis or therapy, it does demonstrate the range of toxic mechanisms and assists in predicting which classes of chemical may lead to toxicity. Benzalkonium chloride, for example, disrupts cell walls by emulsifying membrane lipids, and hence both its effectiveness as a preservative and its toxicity to mammalian cells, as lipids are a component common to all cell walls. It is typically used in concentrations of $0.004-0.01 \%$. Benzalkonium side 
effects include corneal vascularisation at high concentrations $(0.1 \%)$, papillary conjunctivitis, punctate keratopathy, decreased epithelial microvilli reducing corneal wetting, it inhibits cell motility and healing, binds to soft contact lenses enhancing toxicity, causes allergic blepharoconjunctivitis, and has been associated with drug-induced pemphigoid. ${ }^{1}$

Subclinical drug-induced scarring may occur without clinical inflammation, or clinically apparent scarring, in glaucoma patients on long-term miotic and nonmiotic therapy, such as phospholine iodide, pilocarpine, guanethidine, and adrenaline, although all topical antiglaucoma medications have to some degree been implicated. ${ }^{12}$ While some shortening of the conjunctival fornix occurs with age, this is increased by topical therapy. ${ }^{10}$ In these patients, fibroblasts, macrophages, lymphocytes, and mast cells are present in the superior conjunctival substantia propria ${ }^{11}$ and there is a loss of goblet cells in the epithelium.

There are also several well-defined toxic clinical disorders associated with a papillary conjunctival response: clinical scarring without progression (pseudopemphigoid) after exposure to toxic medications, and clinical scarring which is progressive after withdrawal of the drug (known as drug-induced ocular cicatricial pemphigoid). Pseudopemphigoid is characterised by clinical inflammation, persistent epithelial defects, and conjunctival scarring, which is nonprogressive after withdrawal of the precipitating medications. It has been linked with the same range of glaucoma medications that are associated with the subclinical scarring already described. The only systemic medication that has been associated with conjunctival cicatrisation is practolol. Practolol may also cause corneal opacities and rapid corneal melting, as well as nonocular reactions including secretory otitis media, sclerosing peritonitis, and a psoriaform rash. A pemphigus-like antibody, showing intercellular binding to epithelial tissue, and an antinuclear antibody have been demonstrated in the serum of patients taking practolol, but these antibodies appear not to be associated with signs of tissue damage and both may be epiphenomena. ${ }^{12,13}$

Drug-induced ocular cicatricial pemphigoid (OCP) was first described in $1976^{14}$ with two unilateral cases of progressive OCP in patients on topical phospholine iodide, one with immunostaining for IgG at basement membrane. Other cases with definite progression of disease have been described ${ }^{15,16}$ and are related to use of pilocarpine, IDU, and epinephrine. The clinical course, histopathological findings, and response to immunosuppressive therapy in patients with this disease are indistinguishable from those with classical OCP. However, unlike classical OCP, drug-induced OCP may be unilateral secondary to unilateral drug exposure, as in unilateral glaucoma, or herpes keratitis.

Toxic follicular reactions, with or without inflammation, may be associated with pseudodendritic or geographic ulcers and punctal stenosis. Offending drugs include atropine, miotics, epinephrine, and antivirals. Nonprogressive scarring may also occur (pseudotrachoma), but is rare, and is probably the

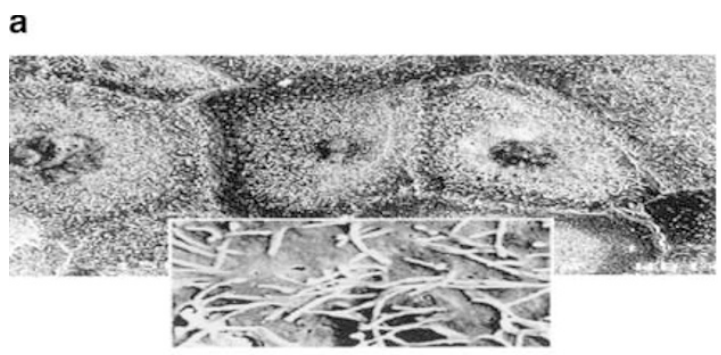

b

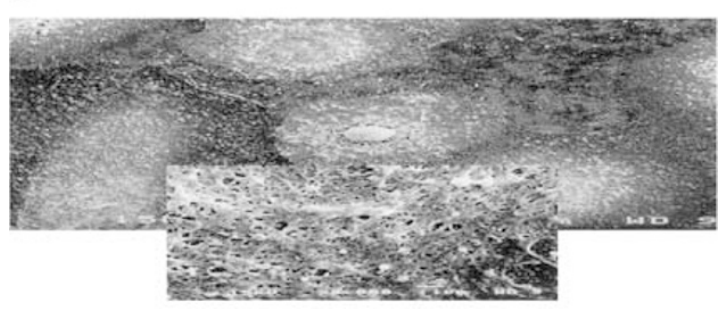

C

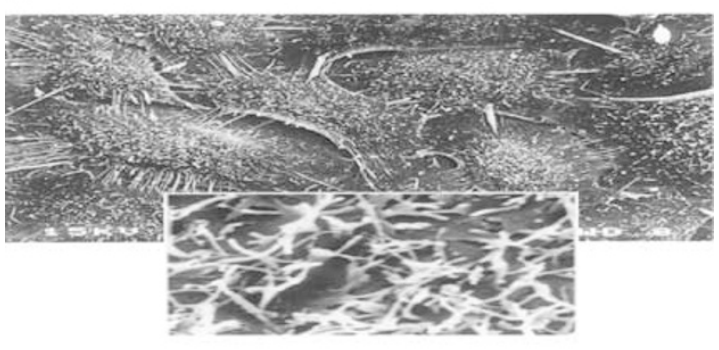

d

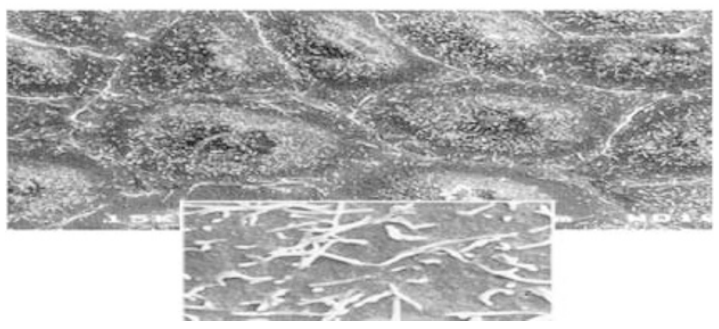

Figure 1 Epithelial cell morphology after different incubation periods for hypromellose (HPMC) preserved with $0.01 \%$ benzalkonium chloride (BAK) and unpreserved 50\% serum: (a) HPMC with $0.01 \%$ BAK after 10 min incubation; (b) HPMC with $0.01 \%$ BAK after $6 \mathrm{~h}$ incubation showing severe loss of microvilli and porosity of the cell membranes; (c) $50 \%$ serum after $10 \mathrm{~min}$ incubation; (d) $50 \%$ serum after $6 \mathrm{~h}$ incubation showing maintenance of microvilli and no membrane porosity. 
consequence of prolonged or severe toxic follicular conjunctivitis ${ }^{1}$ thought to be the result of the mitogenic effect of these drugs.

\section{Predicting toxic reactions}

Drug information sheets give global data about many, but not all, toxicity reactions. The global data are not very helpful, as the probability for the development of reactions is not given even if known. Toxic responses are often because of the preservatives (such as benzalkonium) or the excipients (such as EDTA) rather than the drugs, and drug information sheets often fail to supply relevant information regarding these. The effects of individual components may be established from pharmaceutical references and from those on side effects, for example Grants toxicology, ${ }^{1}$ Martindale, ${ }^{17}$ and the British National Formulary. ${ }^{18}$ Easily accessible data on the probability for the development of an adverse reaction, or an index of toxicity, is not readily available from these sources either, and prediction of problems currently depends largely on clinical awareness and expertise.

\section{Assessment of toxicity}

Some toxic reactions are idiosyncratic and cannot be evaluated except by clinical methods. However, for drugs with short-term and predictable toxic effects, the availability of an index of toxicity, which we could use to compare all topical therapies, would assist in the management of topical therapy. The tests that are currently available are the Draize test in rabbits, which is an accepted part of the development of topical ophthalmic preparations, and in vitro tests on human cell cultures, which are being developed but are not yet accepted as toxicity tests for the development of new drugs. ${ }^{19}$ These tests include ATP assays for cell viability, scanning EM, and vital staining.
The Draize test is used to screen for toxicity at an early stage of drug development. The scoring system grades corneal opacity and the area of corneal involvement, iris inflammation and conjunctival redness, chemosis, and discharge. ${ }^{19}$ It has had a role in screening out drugs having severe toxicity for mammalian ocular tissues but cannot identify the more subtle effects on human ocular epithelia after drug exposure.

Alternatively, the in vitro tests on human cell cultures may be more precise indicators of the potential for toxic reactions in humans, and can be used to create a toxicity index for comparison between drugs. The relevance of such in vitro toxicity data is sometimes questioned because a cultured ocular surface lacks the complex physical and molecular interactions of the tear film and ocular surface in vivo. However, for dry eyes, with an already compromised tear film, in vitro testing may parallel the in vivo environment more closely, allowing test results to be extrapolated to the clinical situation. In vitro tests are not yet accepted for the licensing of drugs although they are in development for this. An example that demonstrates the range of available in vitro tests, and shows good correlation with clinical findings, is from a study of the ocular toxicity of a range of pharmaceutical and physiological tear substitutes on in vitro corneal cell cultures. ${ }^{20}$

This study evaluated hypromellose with and without preservative, saliva, and serum. These tear replacements were chosen because of recent clinical studies on submandibular gland transplantation and autologous serum drops for the management of the advanced dry eye. Three measures of toxicity were used. Epithelial cell microvillous density was evaluated by scanning electron microscopy (see Figure 1), cell membrane permeability and intracellular esterase activity by staining with fluorescent calcein-AM/ethidium-homodimer (see Figure 2), and cellular ATP was quantified using a luciferin-luciferase-based assay (see Figure 3).

The results of all three in vitro tests correlated both with each other and with what was expected following
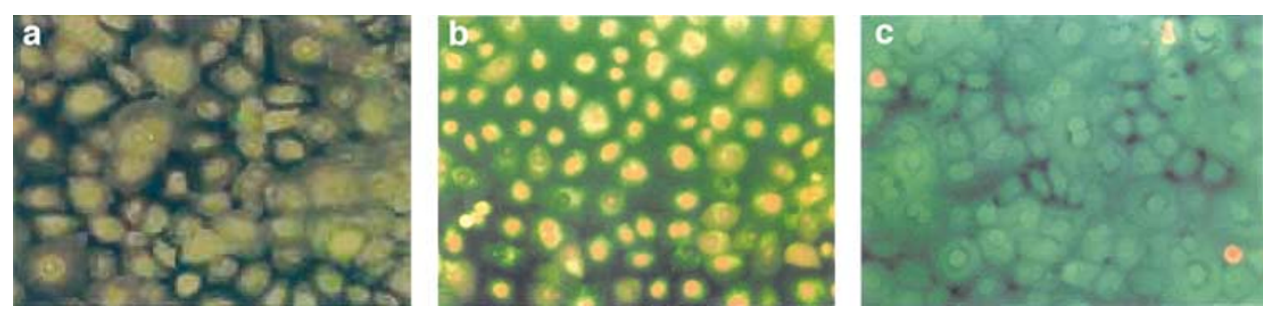

Figure 2 Calcein-AM/ethidium-homodimer fluorescent viability stain for tear substitutes, brown stain indicating cell membrane permeability and green stain for intracellular esterase. $X$ hours exposure. (a) Hypromellose preserved with $0.01 \%$ benzalkonium showing dead cells having loss of cell membrane permeability and loss of intracellular esterase. (b) Unpreserved hypromellose showing more viable cells than in (a) with limited membrane permeability and substantial intracellular esterase. (c) $50 \%$ serum showing viable cells. 
the clinical studies. The ATP assay was the most sensitive. The results of all three tests can be scored to give a toxicity index (Table 3 ) in which toxicity can be scored and ranked. This approach to the evaluation of topical ocular therapy can give useful comparative data for clinicians when trying to evaluate the probability of
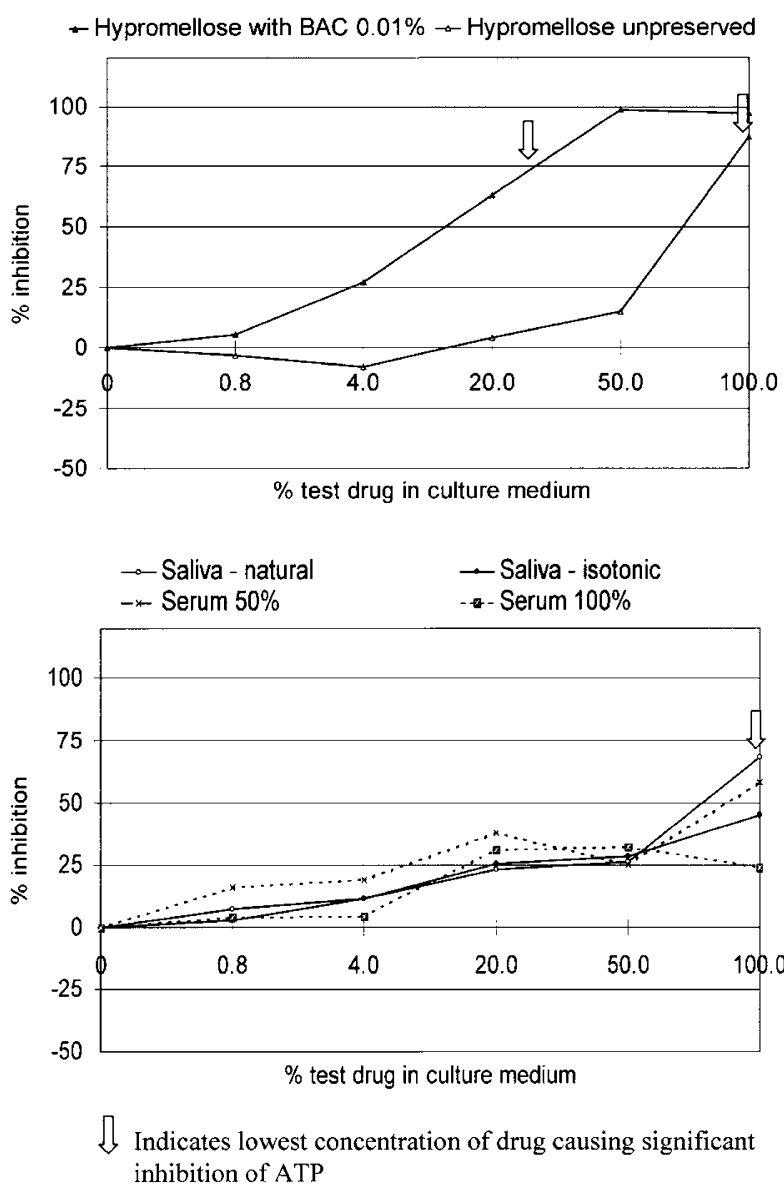

Figure 3 Toxicity as inhibition of cellular ATP in dose-response assays, after $2 \mathrm{~h}$ incubation, for different tear substitutes. ${ }^{20}$ the development of toxic reactions either before the institution of therapy or as the cause of an exacerbation of keratoconjunctivitis in patients with existing ocular surface disease.

\section{Treatment}

The potential for drug toxicity is always present and must be included in the differential diagnosis of all cases with exacerbations of, or poorly controlled, ocular surface disease. As the clinical signs are usually so nonspecific, a diagnosis can only be confirmed by assessing the effect of withdrawing medications where possible. Less toxic or oral substitutes may be prescribed and many patients can have all medication withdrawn. For the latter group, 'comfort' drops may be required for which nonpreserved isotonic saline, balanced salt solution, or nonpreserved artificial tears can be used. These rarely cause a toxic response, although excipients such as EDTA may occasionally cause an adverse response, and clinicians must be aware of all the components of any topical drop including these excipients. The time for a response to withdrawal of toxic drugs is often prolonged: usually 2 weeks (up to 6) to improve and 4 weeks (up to 12) to resolve. Persistent defect, once developed, may fail to respond to drug withdrawal alone. Factitious injury is rare and, because the diagnosis is so difficult to confirm and has such serious implications for the patient, it should only be considered when all other diagnoses and treatment options have been considered. Easily available indices of toxicity would be of value in identification of potential causes.

\section{Prevention}

Prevention demands a high level of awareness of the potential for toxic keratoconjunctivitis. Avoiding the use

Table 3 Toxicity index

\begin{tabular}{|c|c|c|c|c|c|}
\hline \multirow[t]{2}{*}{ Rank and test drug } & \multirow{2}{*}{$\begin{array}{l}\text { SEM of } \\
\text { microvilli }\end{array}$} & \multicolumn{2}{|c|}{ Fluorescent viability staining } & \multirow{2}{*}{$\begin{array}{l}\text { ATP } \\
\text { assay }\end{array}$} & \multirow[t]{2}{*}{ Sum } \\
\hline & & $\begin{array}{l}\text { Cell membrane } \\
\text { impermeability }\end{array}$ & $\begin{array}{c}\text { Loss of esterase } \\
\text { activity }\end{array}$ & & \\
\hline 1. Serum $100 \%$ & NT & 1 & 0 & 1 & 2 \\
\hline 2. Serum $50 \%$ with chloramphenicol & 0 & 1 & 0 & 2 & 3 \\
\hline 3. Saliva-isotonic & 1 & 3 & 0 & 2 & 6 \\
\hline 4. Saliva-natural & 2 & 3 & 1 & 3 & 9 \\
\hline 5. Hypromellose unpreserved & 2 & 4 & 0 & 3 & 9 \\
\hline 6. Hypromellose with benzalkonium $0.01 \%$ & 3 & 4 & 4 & 4 & 15 \\
\hline
\end{tabular}

Ranking of cytotoxicity (in increasing order) for dry eye preparations after incubation with 100\% test drug in the assays shown in Figures 1-3: for scanning electron microscopy (SEM), scores are $0=$ microvilli as in culture medium, $1=$ mild, $2=$ moderate, $3=$ severe loss of microvilli, NT=not tested; for the viability staining and ATP assay, scores are $0=$ no difference from culture medium, $1=$ mild, $2=$ moderate, $3=$ severe toxicity, $4=$ no difference from maximum inhibitor (benzalkonium chloride $0.1 \%$ ). The sum gives the index of toxicity with higher scores indicating increased toxicity. 
of preserved medications or those known to be toxic (ie aminoglycosides, some glaucoma medications, and antivirals) in high-risk cases (chronic disease, dry eyes, and patients on multiple topical therapies) will reduce the frequency of the problem. Treatment without a working diagnosis, or the identification of therapeutic aims, may lead to toxicity because criteria for treatment success are less likely to have been established leading to prolonged therapy. Polypharmacy must be reviewed to keep it to a minimum. Drug and preservative side effects should be understood for each drug that is prescribed so that toxicity, and allergy, are included in the risk/ benefit assessment for topical therapy.

\section{Acknowledgements}

I thank Ms Si Rauz and Dr G Geerling for their critique of the manuscript.

\section{References}

1 Grant WM. Toxicology of the Eye. Charles C Thomas: Springfield, IL, 1986.

2 Duke-Elder S, McFaul PA. Injuries (Part 2). Non-Mechanical Injuries, Vol XIV, System of Ophthalmology. Henry Kimpton: London, 1972.

3 Parsons MR. Factitious keratoconjunctivitis. In: Krachmer JH, Mannis MJ, Holland EJ (eds). Cornea. Vol II. Corneal and External Disease: Clinical Diagnosis and Management, Chap 106. Mosby: St Louis, MO, 1997.

4 Wilson FM. Adverse external ocular effects of topical ophthalmic therapy: an epidemiological, laboratory and clinical study. Trans Am Ophthalmol Soc 1983; 81: 854-965.

5 Wilson FM. Adverse external ocular effects of topical ophthalmic medications. Surv Ophthalmol 1979; 24: 57-88.

6 Rocha G, Brunette I, Francois MLe. Severe toxic keratopathy secondary to topical anaesthetic abuse. Can J Ophthalmol 1995; 30: 198-202
7 Wilson-Holt N, Dart JKG. Thiomersal keratoconjunctivitis, frequency, clinical spectrum and diagnosis. Eye 1989; 3: 581-587.

8 McCulley JP, Moore MB, Martoba A. Mucous fishing syndrome. Ophthalmology 1985; 92: 1262-1265.

9 Trope GE, Jay JL, Dudgeon J, Woodruff G. Self inflicted corneal injuries in children with congenital corneal anaesthesia. Br J Ophthalmol 1985; 69: 551-554.

10 Schwab IR, Linberg JV, Gioia VM, Benson WH, Chao GM. Foreshortening of the inferior conjunctival fornix associated with chronic glaucoma medications. Ophthalmology 1992; 99: 197-201.

11 Broadway DC, Grierson I, O'Brien C, Hitchings RA. Adverse effects of topical antiglaucoma medication. I. The conjunctival cell profile. Arch Ophthamol 1994; 112: 1437-1445.

12 Wright P. Cicatrising conjunctivitis. Trans Ophthalmol Soc UK 1986; 105: 1-17.

13 Amos HE, Lake BG, Artis J. Possible role of antibody specific for a practolol metabolite in the pathogenesis of oculomucocutaneous syndrome. Br Med J 1978; 1: 402-404.

14 Patten JT, Cavanaugh D, Allansmith MR. Induced ocular pseudopemphigoid. Am J Ophthalmol 1976; 82: 272-276.

15 Fiore PM, Jacobs IH, Goldberg DB. Drug induced pemphigoid. A spectrum of diseases. Arch Ophthalmol 1987; 105: 1660-1663.

16 Pouliquen Y, Patey A, Foster CS, Goishot L, Salvodelli M. Drug induced cicatricial pemphigoid affecting the conjunctiva. Light and electron microscopic features. Ophthalmology 1986; 93: 775-783.

17 Martindale. In: The Extra Pharmacopoeia, Reynolds JEF (ed). 29th edn. The Pharmaceutical Press: London, 1989.

18 British National Formulary 39 The British Medical Association \& The Royal Pharmaceutical Society of Great Britain, London, March 2000.

19 Geerling G, Baatz J, Harder D, Muller G, Reimer K, Rudolph P, Kramer A . Local tolerance. In: Kramer A, Behrens-Baumann W (eds). Antiseptic Prophylaxis and Therapy in Ocular Infections, Chap 4.2. Karger: Basel. Dev Ophthalmol 2002; 33: 32-56.

20 Geerling G, Daniels JT, Dart JKG, Cree IA, Khaw PT. Toxicity of natural tear substitutes in a fully defined culture model of human corneal epithelial cells. Invest Ophthalmol Vis Sci 2001; 42: 948-956. 